\title{
Discrimination between Glioblastoma and Solitary Brain Metastasis: Comparison of Inflow-Based Vascular-Space- Occupancy and Dynamic Susceptibility Contrast MR Imaging
}

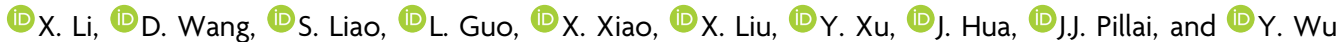

\begin{abstract}
BACKGROUND AND PURPOSE: Accurate differentiation between glioblastoma and solitary brain metastasis is of vital importance clinically. This study aimed to investigate the potential value of the inflow-based vascular-space-occupancy MR imaging technique, which has no need for an exogenous contrast agent, in differentiating glioblastoma and solitary brain metastasis and to compare it with DSC MR imaging.
\end{abstract}

MATERIALS AND METHODS: Twenty patients with glioblastoma and 22 patients with solitary brain metastasis underwent inflowbased vascular-space-occupancy and DSC MR imaging with a $3 \mathrm{~T}$ clinical scanner. Two neuroradiologists independently measured the maximum inflow-based vascular-space-occupancy-derived arteriolar CBV and DSC-derived CBV values in intratumoral regions and peritumoral T2-hyperintense regions, which were normalized to the contralateral white matter (relative arteriolar CBV and relative CBV, inflow-based vascular-space-occupancy relative arteriolar CBV, and DSC-relative CBV). The intraclass correlation coefficient, Student $t$ test, or Mann-Whitney $U$ test and receiver operating characteristic analysis were performed.

RESULTS: All parameters of both regions had good or excellent interobserver reliability $(0.74 \sim 0.89)$. In peritumoral T2-hyperintese regions, DSC-relative CBV $(P<.001)$, inflow-based vascular-space-occupancy arteriolar CBV $(P=.001)$, and relative arteriolar CBV $(P=$ .005) were significantly higher in glioblastoma than in solitary brain metastasis, with areas under the curve of $0.94,0.83$, and 0.72 for discrimination, respectively. In the intratumoral region, both inflow-based vascular-space-occupancy arteriolar CBV and relative arteriolar CBV were significantly higher in glioblastoma than in solitary brain metastasis (both $P<.001$ ), with areas under the curve of 0.91 and 0.90 , respectively. Intratumoral DSC-relative CBV showed no significant difference $(P=.616)$ between the 2 groups.

CONCLUSIONS: Inflow-based vascular-space-occupancy has the potential to discriminate glioblastoma from solitary brain metastasis, especially in the intratumoral region.

ABBREVIATIONS: $\mathrm{AUC}=$ area under the curve; $\mathrm{CBV}$ a $=$ arteriolar CBV; GBM = glioblastoma; iVASO = inflow-based vascular-space-occupancy; rCBV $=$ relative $\mathrm{CBV} ; \mathrm{rCBVa}=$ relative arteriolar $\mathrm{CBV} ; \mathrm{PTH}=$ peritumoral $\mathrm{T} 2$-hyperintesity region; $\mathrm{SBM}=$ solitary brain metastasis

G lioblastoma (GBM) accounts for 40\% 50\% of all primary malignant brain tumors in adults. Brain metastases are the most common complication of systemic cancer, and half of them

Received July 23, 2019; accepted after revision February 03, 2020.

From the Department of Medical Imaging (X. Li, S.L., L.G., X.X., X. Liu, Y.X., Y.W.), Nanfang Hospital, Southern Medical University, Guangzhou, P.R. China; School of Biomedical Engineering (D.W.), Shanghai Jiao Tong University, Shanghai, P.R. China; Division of CT and MR, Radiology Department (S.L.), First Affiliated Hospital of Gannan Medical University, Ganzhou, P.R. China; Neurosection, Division of MR Research (J.H.); Division of Neuroradiology (J.P.); Russell H. Morgan Department of Radiology and Radiological Science and Department of Neurosurgery (J.P.), Johns Hopkins University School of Medicine, Baltimore, Maryland; and F.M. Kirby Research Center for Functional Brain Imaging (J.H.), Kennedy Krieger Institute, Baltimore, Maryland.

Xiaodan Li and Danni Wang contributed equally to this study.

This study has received funding from the Natural Science Foundation of Guangdong Province, China (grant No. S201301005689), the Science and Technology Program of Guangzhou, China (grant No. 201707010003), and the Special Foundation of President of Nanfang Hospital, Southern Medical University (grant No. 2016B026). are solitary at diagnosis. ${ }^{1}$ It is clinically important to distinguish GBM from solitary brain metastasis (SBM) because of the vast differences in these 2 entities with regard to tumor staging, treatment approach, and clinical outcomes. ${ }^{2-4}$ Structural gadoliniumenhanced MR imaging is the preferred imaging examination for brain tumors, but with a limited capacity to differentiate GBM and SBM. They share similar imaging features, such as extensive

Abstract previously presented orally at: Annual Meeting and Exhibition of the International Society for Magnetic Resonance in Medicine, May 11-16, 2019; Montreal, Quebec, Canada. Please address correspondence to Yuankui Wu, MD, Department of Medical Imaging, Nanfang Hospital, Southern Medical University, No. 1838 Guangzhou Avenue North, Guangzhou, P.R. China; e-mail: ripleyor@126.com

-- Indicates open access to non-subscribers at www.ajnr.org

$\equiv$ Indicates article with supplemental on-line tables.

http://dx.doi.org/10.3174/ajnr.A6466 
edema and ring-enhancement, which is a great challenge in clinical practice. ${ }^{5-7}$

Many studies have demonstrated that PWI is a promising technique to discriminate GBM from SBM, due to its capability to disclose the differences between them in angiogenesis and vascularity. ${ }^{8,9}$ In particular, DSC MR imaging is the most robust perfusion technique to perform such a task. ${ }^{10,11}$ However, most studies found that DSC-derived relative CBV (rCBV) in intratumoral regions does not permit reliable differentiation between high-grade gliomas and metastases, ${ }^{1,6,12-15}$ which was thought to be related to contrast leakage from tumor vessels and, consequently, unreliable estimation of CBV. ${ }^{10,16,17}$ rCBV measured in peritumoral regions may be effective in this regard, but this method inherently has some major disadvantages due to indefinite tumoral boundary and various definitions of the peritumoral area. ${ }^{15,18}$ Besides, the administration of exogenous contrast agents required for DSC raises concerns about the adverse effects of gadolinium, especially the deposits in brain, even using macrocyclic gadolinium-based contrast agents. ${ }^{19-22}$

Inflow-based vascular-space-occupancy (iVASO) is a completely noninvasive perfusion method that does not involve administration of an exogenous contrast agent. ${ }^{23}$ Instead, proton spins in the water molecules in blood are exploited as an endogenous contrast agent by applying spatially selective radiofrequency inversion pulses. ${ }^{24}$ iVASO emphasizes the perfusion blood volume in arteries and arterioles. The absolute CBV of pial arteries and precapillary arterioles (arteriolar CBV [CBVa]) can be calculated from the different signals between a scan with arterial blood signal selectively zeroed out (nulled) and a control scan without blood nulling. ${ }^{24,25}$ Notably, previous studies have demonstrated that pial arteries and arterioles are the most sensitive vessels to respond via adaptive hemodynamic adjustment to changes in cerebral metabolism status in the human body. ${ }^{26-28}$ According to recent studies, CBVa measured with iVASO MR imaging (iVASO-CBVa) has proved sensitive in disclosing microvascular abnormalities in the early stage of some mental and cognitive disorders, such as Huntington disease, Alzheimer disease, and schizophrenia. ${ }^{29-31}$ Furthermore, a previous study has demonstrated that iVASO-CBVa is strongly correlated with glioma grades and might be superior to DSC-derived $\mathrm{rCBV}^{32}$ Therefore, we hypothesized that iVASO can distinguish GBM from SBM. To validate this hypothesis, we performed iVASO MR imaging on patients with GBM or SBM on a clinical 3T MR imaging scanner.

\section{MATERIALS AND METHODS Study Participants}

This retrospectively reviewed study was prospectively controlled and conducted between December 2015 and March 2017. All examinations were performed in accordance with institutional (Nanfang Hospital Southern Medical University) review board guidelines with an approved study protocol. Inclusion criteria were as follows: 1) patients with a single, solitary enhancing brain mass with a clinical question of SBM versus GBM; 2) pretreatment acquisition of a 3T MR imaging brain tumor protocol, including structural MR imaging, iVASO, and DSC; and 3) the mass pathologically confirmed by stereotactic biopsy or surgical sample within 2 weeks after MR imaging. Ten patients were excluded ( 4 for obvious movement artifacts, 4 for susceptibility artifacts hampering ROI placement, 2 for small lesions greatly influenced by partial volume averaging effect). The remaining 20 patients with GBM and 22 with SBM were eventually included in the study.

\section{MR Imaging Acquisition}

MR imaging examinations were implemented with a clinical 3T imaging unit (Achieva 3T; Philips Healthcare, Best, the Netherlands) equipped with an 8-channel head coil. DSC, iVASO, and structural MR imaging were performed for each subject in the same scan session.

The structural MR imaging protocol included an axial FLAIR scan $(\mathrm{TR} / \mathrm{TI} / \mathrm{TE}=11,000 / 2200 / 125 \mathrm{~ms}$, voxel $=0.7 \times 0.7 \times 6$ $\mathrm{mm}^{3}, 20$ slices), T2WI (TR/TE $=3000 / 80 \mathrm{~ms}$, voxel $=0.5 \times$ $0.7 \times 6 \mathrm{~mm}^{3}, 20$ slices $)$, and T1WI (TR/TE $=2000 / 20 \mathrm{~ms}$, voxel $=$ $0.5 \times 0.9 \times 6 \mathrm{~mm}^{3}, 20$ slices) (detailed in On-line Table 1). Contrast-enhanced fat-suppressed T1WI (TR/TE $=297 / 4.6 \mathrm{~ms}$, voxel $=0.5 \times 0.7 \times 6 \mathrm{~mm}^{3}, 20$ slices) was obtained after DSC.

$3 \mathrm{D}$ iVASO was performed before contrast agent administration. Parameters for the iVASO pulse sequence were the following: $\mathrm{TR} / \mathrm{TI}=5000 / 1040,3100 / 862,2500 / 756,2000 / 641,1700 /$ $558,1300 / 430 \mathrm{~ms} ; 3 \mathrm{D}$ gradient spin-echo readout $(\mathrm{TE}=10 \mathrm{~ms}$; voxel $=2.5 \times 2.5 \times 6 \mathrm{~mm}^{3}, 14$ slices); parallel imaging acceleration (sensitivity encoding) $=2 \times 2$; crusher gradients of $b=0.3 \mathrm{~s} /$ $\mathrm{mm}^{2}$ and $\mathrm{Venc}=10 \mathrm{~cm} / \mathrm{s}$ along the $\mathrm{z}$-direction. A reference scan ( $\mathrm{TR}=20$ seconds, other parameters identical) was obtained to determine the scaling factor M0 in iVASO images so that absolute CBVa values could be calculated. The total duration of all iVASO-related scans combined was 7 minutes for each patient.

DSC perfusion images were acquired immediately after contrast agent injection with fast-field echo, echo-planar imaging, TR/ $\mathrm{TE}=1700 / 40 \mathrm{~ms}, \mathrm{FOV}=210 \times 210 \mathrm{~mm}$, pixel $=2.3 \times 2.3 \mathrm{~mm}$, matrix $=90 \times 90$, thickness/gap $=6 / 0 \mathrm{~mm}, 20$ slices, 60 dynamics, flip angle $=75^{\circ}$. Contrast agent (gadodiamide, Omniscan; GE Healthcare, Piscataway, New Jersey) was administered at a dose of $0.2 \mathrm{mmol} / \mathrm{kg}$ and a rate of $4.5 \mathrm{~mL} / \mathrm{s}$, using a power injector (Spectris Solaris EP; MedRad, Indianola, Pennsylvania) through the antecubital vein, followed by a $20-\mathrm{mL}$ sterile saline flush at the same rate. The total acquisition time of DSC was 1 minute 42 seconds.

\section{MR Imaging Analysis}

All iVASO data were preprocessed using the Statistical Parametric Mapping software package (Version 8; http://www.fil.ion. ucl.ac.uk/spm/software/spm12). The iVASO images were analyzed with in-house routines programmed in Matlab (MathWorks, Natick, Massachusetts), which were used for a previously published report. ${ }^{32}$ DSC images were processed on an Advantage Workstation using FuncTool (Version 4.6; GE Healthcare) to obtain CBV maps.

Color-coded iVASO-CBVa and DSC-CBV maps were generated, respectively. The region of maximal abnormality of each parameter within the lesion volume (hotspot) was determined via visual inspection. This methodology was demonstrated to provide the most optimal interobserver and intraobserver reproducibility. ${ }^{33}$ Four ROIs of about 20 pixels were carefully placed on the hotspots, 


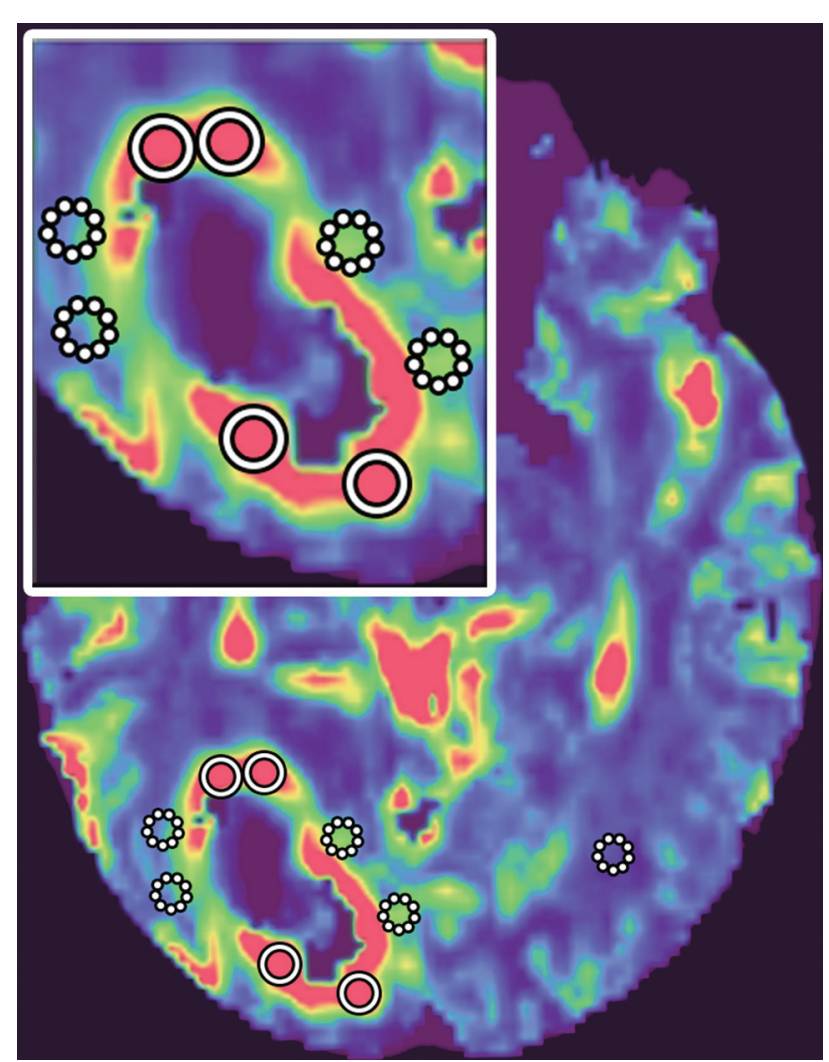

FIG 1. ROI placements. Four to six ROls were drawn in both intratumoral (circle with solid line) and peritumoral (circle with dotted line) regions, and the maximum value was recorded. Also, an ROI in the contralateral white matter (circle with dotted line) was chosen as a reference. The insert is the magnification of lesion area.

respectively, in the intratumoral region and the peritumoral T2hyperintense region $(\mathrm{PTH})$, to obtain the maximum iVASO-CBVa and CBV of each region. The PTH was defined as the T2-hyperintense region within $1 \mathrm{~cm}$ around the enhancing tumor. ${ }^{13}$ ROIs were drawn in the contralateral white matter as references for normalization (iVASO-rCBVa and DSC-rCBV) (Fig 1). All ROIs were placed independently by 2 blinded experienced neuroradiologists (X. Li and $\mathrm{Y}$. Wu, with 5 and 12 years of experience, respectively). The measurement results of the 2 radiologists were used to assess the interobserver reliability. The average of the 2 measurement results was used for further statistical analysis.

\section{Statistical Analysis}

Statistical analysis was performed using SPSS Statistics 22.0 (IBM, Armonk, New York). Interobserver reliability of the parameters between the 2 neuroradiologists was assessed by the intraclass correlation coefficient with $95 \%$ confidence intervals. Intraclass correlation coefficient values of $\leq 0.40$, between 0.41 and 0.60 , between 0.61 and 0.80 , and $\geq 0.81$ were interpreted as poor, moderate, good, and excellent reliability, respectively. iVASO-CBVa, rCBVa, and DSC-rCBV in intratumoral and peritumoral regions were correlated with each other by calculating Pearson correlation coefficients. The Shapiro-Wilk test was used to assess the normality of data distribution. Comparisons between the GBM and SBM groups were performed using the Student $t$ test or Mann-Whitney $U$ test accordingly. Receiver operating characteristic and area under the curve (AUC) were used to assess the diagnostic value of parameters for discrimination. An area under the receiver operating characteristic curve greater than 0.90 was considered excellent; $0.80-0.90$ was considered good; $0.70-0.80$ was considered fair; $0.60-0.70$ was considered poor; and $<0.50$ was considered a failure. The cutoff value was established by maximizing the Youden index (Youden index $=$ sensitivity + specificity -1). A statistical significance of a $P$ value $<.05$ was used.

\section{RESULTS}

Twenty GBMs (16 men and 4 women, with a mean age of 46.1 years; range, $18-62$ years), which included 19 patients without the isocitrate dehydrogenase 1 (IDH1) mutation and 1 with the IDH1 mutation, and 22 SBMs ( 13 men and 9 women, with a mean age of 56.6 years; range, $44-65$ years), which included 18 patients with primary non-small cell lung adenocarcinoma, 2 with breast adenocarcinoma, 1 with prostate cancer, and 1 with hepatocellular carcinoma, finally met all of our inclusion and exclusion criteria. Among them, 2 patients with GBMs and 17 with SBMs were diagnosed on the basis of stereotactic biopsy samples, and the conditions of the others were confirmed by gross total resection.

The interobserver reliability was excellent for iVASO-CBVa in PTH (intraclass correlation coefficient $=0.82$ ) and was good for DSC-rCBV (intraclass correlation coefficient $=0.80$ ) and iVASO-rCBVa (intraclass correlation coefficient $=0.74$ ). In the intratumoral region, all the parameters demonstrated excellent reliability (intraclass correlation coefficient $=0.86 \sim 0.89$ ).

The results of correlation analysis are given in On-line Table 2. In the intratumoral region, no substantial correlation was observed between iVASO-CBVa or rCBVa and DSC-rCBV $(P=.23$ and .18$)$, while in the peritumoral region, a mild correlation was observed between iVASO-CBVa or rCBVa and DSC$\operatorname{rCBV}(P<.001)$.

Perfusion values are plotted in Fig 2. In intratumoral regions, both iVASO-CBVa and $\mathrm{rCBVa}$ were significantly higher in patients with GBM than in those with SBM $(P=.001$ and .005 , respectively), while DSC-rCBV showed no significant difference between them $(P=.616)$. In PTH, DSC-rCBV, iVASO-CBVa, and $\mathrm{rCBVa}$ revealed higher values in GBM than in SBM $(P<.001)$. Representative cases including iVASO and DSC perfusion MR images are shown in Fig 3.

The results of receiver operating characteristic analysis of each parameter for differentiating GBM and SBM are listed in the Table and plotted in Fig 4. In intratumoral regions, both iVASOCBVa and rCBVa showed excellent performance, with AUCs of 0.91 and 0.90 , a sensitivity of $80 \%$ and $70 \%$, and a specificity of $100 \%$ and $100 \%$, respectively, which was comparable with that of DSC-rCBV in PTH (AUC, 0.94; sensitivity, 80\%; specificity, 100\%). In PTH, the AUCs of iVASO-CBVa and rCBVa were 0.83 and 0.72 , respectively.

\section{DISCUSSION}

Reliable differentiation between GBM and SBM is of vital clinical importance. Our preliminary study investigated the capacity of 

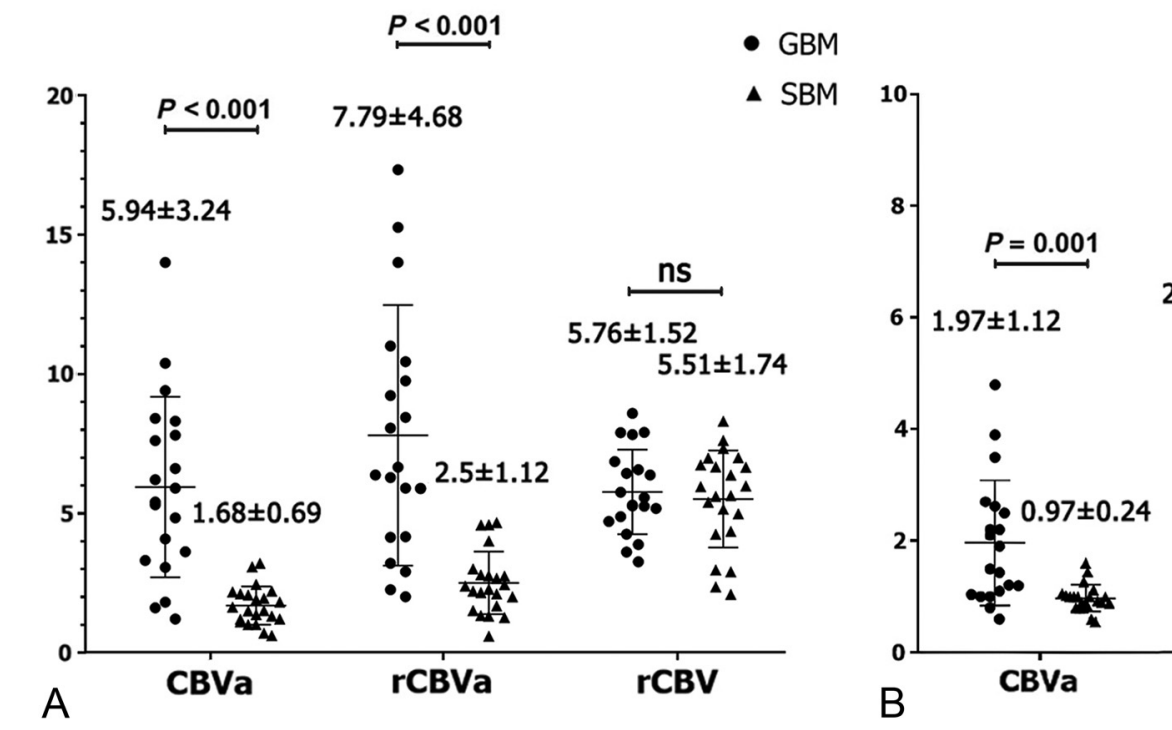

FIG 2. iVASO-CBVa, rCBVa, and DSC-rCBV in the intratumoral region $(A)$ and in peritumoral T2-hyperintense region (B) of glioblastoma and single brain metastasis. Data are presented as mean value \pm SD. Ns indicates not significant.

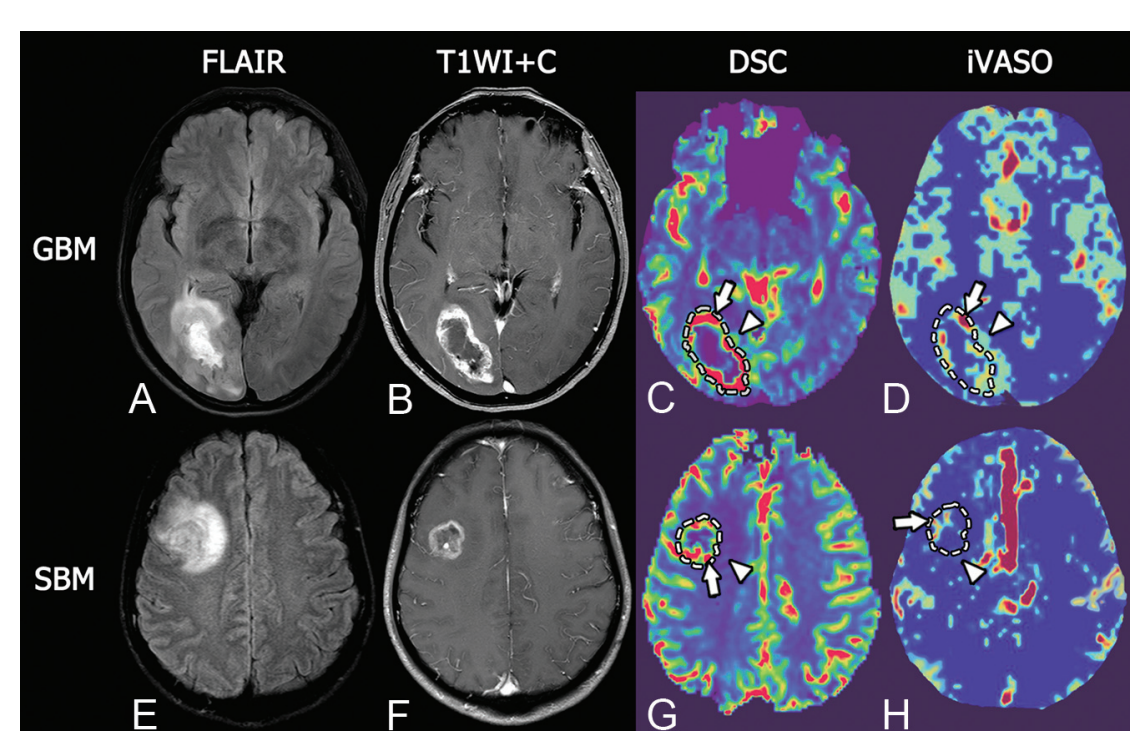

FIG 3. Representative MR images of glioblastoma and single brain metastasis. Upper row, A GBM in a 51-year-old woman. Lower row, SBM in a 46-year-old man. Both GBM and SBM present as hyperintense masses on T2 FLAIR with extensive peritumoral edema ( $A$ and $E$ ) and show a ring-enhancement pattern on fat-suppressed postcontrast TIWI with prominent necrosis in the tumor center ( $B$ and $F$ ). In intratumoral regions, GBM shows maximum DSC-rCBV similar to that of SBM (5.29 versus 4.98, arrows in $C$ and $G$ ), but higher maximum iVASO-CBVa than SBM (5.90) $100 \mathrm{~mL}$ versus $1.30 / 100 \mathrm{~mL}$, arrows in $D$ and $H$ ). In the peritumoral region, GBM shows prominently higher DSC-rCBV and iVASO-CBVa than SBM (DSC-rCBV, 3.11 versus 1.25, arrowheads in C and $G$; iVASO-CBVa, $1.20 / 100 \mathrm{~mL}$ versus $0.55 / 100 \mathrm{~mL}$, arrowheads in $D$ and $H$ ).

obviously different perfusion values in the PTH between these entities. Histopathologically, GBM tends to grow in an invasive manner and extends to the PTH beyond the contrast-enhancing margins. ${ }^{34-39}$ On the contrary, SBM tends to grow in an expansile way, leading to no prominent infiltration of tumor cells in the PTH beyond the area of contrast enhancement. ${ }^{15,35,40}$ However the definition of the tumor boundary is a common issue of controversy within this field. Researchers have defined the tumoral and peritumoral areas in various ways. $1,13,15,41$ For gliomas, the so-called peritumoral regions pathologically consist of benign changes, such as vasogenic edema and inflammatory reaction, as well as infiltration by tumor cells. Besides, the peritumoral edema areas of GBM and metastasis are usually extensive and may include different lobes and even extend to the whole cerebral hemisphere and the contralateral hemisphere. This feature will make drawing the ROI relatively difficult and thus affect the interobserver reliability, ${ }^{42}$ as shown

iVASO MR imaging to differentiate these 2 types of tumors. The results showed that iVASO-derived CBVa and rCBVa in both intratumoral and peritumoral PTH can accurately discriminate GBM and SBM and that DSC-rCBV was powerful for the discrimination between them only in PTH.

Within PTH, DSC-rCBV can accurately discriminate GBM from SBM according to our study, which was concordant with previous reports. ${ }^{5,14,15}$ This finding may be explained by the in our present study in which the interobserver reliability of the peritumoral region $(0.74 \sim 0.82)$ was lower than that of the intratumoral region $(0.86 \sim 0.89)$.

According to most previous studies, the intratumoral region is the mainstream region for measurement. ${ }^{43,44}$ The intratumoral perfusion is closely related to tumor biologic characteristics, gene mutation status, therapeutic response, and prognosis. ${ }^{45-48}$ Unfortunately, most of these studies have demonstrated that 
intratumoral DSC-rCBV was not powerful for differentiating GBM from SBM, ${ }^{1,6,12-15}$ as shown in our study. Of note, this finding does not mean that these 2 types of tumors share the same characteristics of microvasculature. Weber et $\mathrm{al}^{13}$ observed significantly larger microvessel density in GBM than in brain adenocarcinoma metastases. According to the study of Jinnouchi et al, ${ }^{49}$ the capillaries of brain metastasis resemble those from the site of the original systemic cancer and thus have no similarity to the normal brain capillaries and completely lack BBB components. On the other hand, GBM is primary brain tumor and has a blood-brain barrier, albeit a heterogeneous, disrupted one. ${ }^{8,50}$ Lai et $\mathrm{al}^{51}$ and $\mathrm{Fu}$ et $\mathrm{al}^{52}$ reported that the degree of intralesional susceptibility signal was significantly higher in GBM than in SBM. Intralesional susceptibility signal reflects the conglomerates of tumor microvascularity, and the degree of intralesional susceptibility signal showed a significant correlation with the value of maximum DSC-rCBV in the same tumor segments. ${ }^{44,53,54}$ Furthermore, a few investigators reported significant perfusion

\begin{tabular}{|c|c|c|c|c|c|}
\hline Technique/Parameter & AUC & $P$ Value & Cutoff & Se (\%) & Sp (\%) \\
\hline \multicolumn{6}{|l|}{$\begin{array}{l}\text { Intratumoral region } \\
\text { iVASO }\end{array}$} \\
\hline CBVa & 0.91 & $<.001$ & 3.25 & 80.0 & 100.0 \\
\hline rCBVa & 0.90 & $<.001$ & 5.28 & 70.0 & 100.0 \\
\hline \multicolumn{6}{|l|}{ DSC } \\
\hline rCBV & 0.51 & .920 & 3.11 & 100.0 & 18.2 \\
\hline \multicolumn{6}{|l|}{$\begin{array}{l}\text { Peritumoral region } \\
\text { iVASO }\end{array}$} \\
\hline CBVa & 0.83 & $<.001$ & 1.03 & 80.0 & 77.3 \\
\hline rCBVa & 0.72 & .014 & 2.04 & 55.0 & 95.5 \\
\hline \multicolumn{6}{|l|}{ DSC } \\
\hline rCBV & 0.94 & $<.001$ & 2.26 & 80.0 & 100.0 \\
\hline
\end{tabular}

Note: Se indicates sensitivity; Sp, specificity.

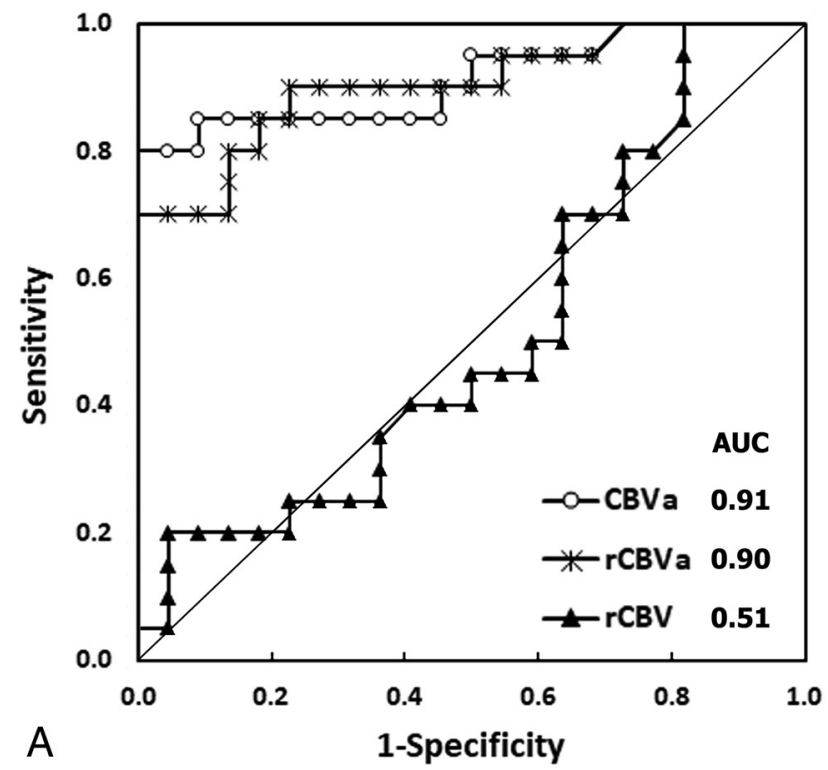

differences between GBM and SBM, using parameters of peak height or percentage signal recovery ${ }^{35,55,56}$ or histogram analysis of $\mathrm{rCBV}{ }^{57}$

In the present study, both iVASO-CBVa and rCBVa accurately differentiated GBM from SBM and outperformed DSCrCBV without leakage correlation via assessment of the intratumoral regions. Interobserver reliability analysis demonstrated poor reliability between iVASO-derived parameters and DSCrCBV in intratumoral regions. This may be mainly due to the different compartments assessed by iVASO and DSC. iVASO is designed to quantify the blood volume of the arterioles, while DSC quantifies the perfusion of the whole microvasculature. ${ }^{23,24}$ Physiologically, arterioles and pial arteries are the most actively regulated blood vessels in the microvasculature. ${ }^{27,58,59}$ They control the cerebral perfusion of the whole microvasculature unit through the contraction and relaxation of smooth-muscle and elastic lamina. ${ }^{60,61}$ Also, there is evidence that generation of arterioles occurs before capillary growth in angiogenesis. ${ }^{62}$ The predominant arterial origin of the iVASO signal was validated in a previous study by measuring the transverse relaxation times $\left(\mathrm{T} 22^{*} / \mathrm{T} 2\right)$ of iVASO difference signals, which are highly oxygenation-level dependent. ${ }^{63}$ Besides, the iVASO signal changes during functional stimulation, such as somatosensory stimulus and forepaw stimulation, preceded the changes in total CBV, ${ }^{24}$ which corresponded to animal studies showing earlier changes in arterioles upon neuronal activation. ${ }^{64,65}$ Therefore, the ability to measure arteriolar CBV separately from the rest of the microvasculature (capillaries and venous vessels) may furnish information that is not obtainable from total CBV measures (ie, DSC$\mathrm{CBV}$ ) and may make the measurement more sensitive in reflecting hemodynamics changes. ${ }^{31}$ Notably, the wall of the arterioles is not permeable to magnetically labeled spin protons. Hence, the CBVa value would not be affected by the disrupted bloodbrain barrier. In contrast, the measurement of DSC-rCBV is

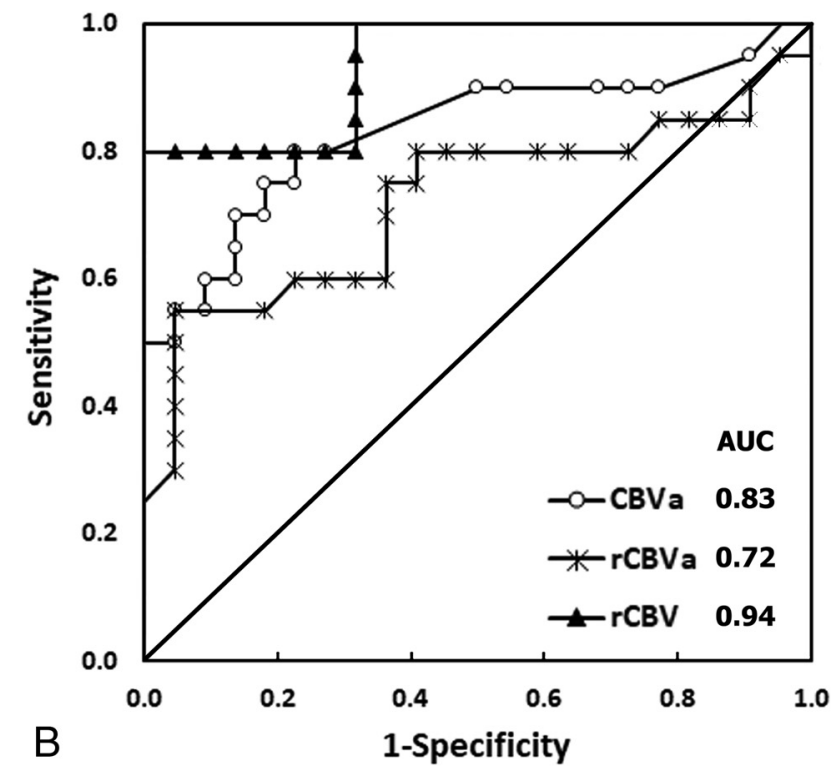

FIG 4. Receiver operating characteristic curves of parameters of iVASO and DSC MR imaging in differentiating glioblastoma and solitary brain metastasis. In the intratumoral region $(A)$, both iVASO-CBVa (AUC $=0.91)$ and $r C B V a(A U C=0.90)$ show higher AUCs than DSC-rCBV (AUC $=$ 0.51). In the PTH (B), the AUC of DSC-rCBV (0.94) is higher than that of iVASO-CBVa (0.83) or rCBVa (0.72). 
remarkably confounded by the disrupted blood-brain barrier. Our results demonstrated significant differences in iVASO-CBVa between GBM and SBM. This finding indicates the difference in the arteriolar compartment between them, which is in line with the results revealed by previous studies. ${ }^{8,13,49-52}$

Most interesting, intratumoral iVASO-CBVa had a diagnostic value approximate to that of iVASO-rCBVa. This finding may suggest that the discrimination between GBM and SBM can be achieved by measuring the perfusion value within the intratumoral region alone, which will enhance the clinical applicability of iVASO, whereas iVASO-CBVa and rCBVa in the PTH showed lower capability than their intratumoral counterparts in distinguishing these 2 groups of tumors. This might be related to the heterogeneity and complexity of the microenvironment in the PTH. Also, according to the theory of iVASO, the measurement of iVASO-CBVa is based on the arterial transit time of gray matter, so it is sensitive to blood flow with a relatively high speed. ${ }^{23}$ However, the arterial transit time in white matter is relatively long, which will reduce the sensitivity of iVASO in quantifying perfusion. ${ }^{24,66,67}$ In contrast, DSC is designed to mainly quantify the capillary bed, so it will not be affected by the relatively slow blood flow in the white matter regions. ${ }^{32}$

DSC MR imaging, the most widely used perfusion MR imaging technique, was recommended as a routine protocol by the 2018 European Guidelines in brain tumor MR scanning. ${ }^{11}$ However, the deposit of exogenous contrast agent of gadolinium is a major issue of public concern. ${ }^{19-22}$ Also, logistically, the inconvenience of the bolus injection of contrast agent in children and elderly patients has limited the application of DSC scanning. ${ }^{32,67}$ Besides, according to most studies, ${ }^{1,12}$ DSC failed to discriminate GBM from SBM via analysis of the intratumoral region, just as shown in our present study. iVASO is a totally noninvasive perfusion technique without the need for exogenous contrast agents. Of note, the actual scan time of iVASO MR imaging is usually several minutes longer than DSC MR imaging. However, one important practical advantage of iVASO is that the perfusion data can be obtained in a flexible manner and can be integrated into a conventional MR imaging examination at any time as long as no contrast agent has been administered. ${ }^{32,67}$

Our study has several limitations. First, the sample size is relatively small. Therefore, a larger cohort study is needed to validate these results in the future. Also, metastases of other different cancer subtypes were not included in our study. Second, we did not use histogram analysis to study the tumor perfusion. Generally, histogram analysis reveals more objective results. However, the additional time-consuming postprocessing involved may lower its clinical practicability. Considering that the hotspot method showed good reliability, we believed that this would not essentially affect our main results. Moreover, we did not apply the leakage-correction analysis method or preload gadolinium-based contrast agent in our DSC protocol to reduce the variance of gadolinium rCBV estimates. ${ }^{68}$ However, a recent DSC study applying preload of contrast agent failed to discriminate these 2 groups of tumors in the intratumoral region. ${ }^{12}$ Moreover, the complexity of the operation and the consumption of more time, which greatly hinder patient compliance and cause motion artifacts, limit the use of leakage-correction and preload strategy in clinical practice. ${ }^{69}$ In addition, it would be better to perform imaging-pathology correlation analysis. However, because the section thickness $(6 \mathrm{~mm})$ of the current iVASO technique is inferior to the requirement for stereotactic biopsy, one-to-one correspondence between the biopsied regions and imaged regions is not possible.

\section{CONCLUSIONS}

This preliminary study demonstrated that iVASO might be useful for discriminating GBM from SBM based on the analysis of either PTH or intratumoral region. Due to its completely noninvasive nature, iVASO might greatly benefit patients with brain tumor in daily clinical practice, especially for elderly populations and those with compromised renal function.

Disclosures: Jay Pillai-UNRELATED: Royalties: Springer Science \& Business Media, Elsevier, Comments: royalties for books published.

\section{REFERENCES}

1. Bauer AH, Erly W, Moser FG, et al. Differentiation of solitary brain metastasis from glioblastoma multiforme: a predictive multiparametric approach using combined MR diffusion and perfusion. Neuroradiology 2015;57:697-703 CrossRef Medline

2. Soffietti R, Abacioglu U, Baumert B, et al. Diagnosis and treatment of brain metastases from solid tumors: guidelines from the European Association of Neuro-Oncology (EANO). Neuro Oncol 2017;19:162-74 CrossRef Medline

3. Ahmed R, Oborski MJ, Hwang M, et al. Malignant gliomas: current perspectives in diagnosis, treatment, and early response assessment using advanced quantitative imaging methods. Cancer Manag Res 2014;6:149-70 CrossRef Medline

4. Giese A, Westphal M. Treatment of malignant glioma: a problem beyond the margins of resection. J Cancer Res Clin Oncol 2001; 127:217-25 CrossRef Medline

5. Lee EJ, Ahn KJ, Lee EK, et al. Potential role of advanced MRI techniques for the peritumoural region in differentiating glioblastoma multiforme and solitary metastatic lesions. Clin Radiol 2013;68: e689-97 CrossRef Medline

6. Server A, Orheim TE, Graff BA, et al. Diagnostic examination performance by using microvascular leakage, cerebral blood volume, and blood flow derived from 3-T dynamic susceptibility-weighted contrast-enhanced perfusion MR imaging in the differentiation of glioblastoma multiforme and brain metastasis. Neuroradiology 2011;53:319-30 CrossRef Medline

7. Maluf FC, DeAngelis LM, Raizer JJ, et al. High-grade gliomas in patients with prior systemic malignancies. Cancer 2002;94:3219-24 CrossRef Medline

8. Wesseling P, Ruiter DJ, Burger PC. Angiogenesis in brain tumors; pathobiological and clinical aspects. J Neurooncol 1997;32:253-65 CrossRef Medline

9. Long DM. Capillary ultrastructure in human metastatic brain tumors. J Neurosurg 1979;51:53-58 CrossRef Medline

10. Cha S, Knopp EA, Johnson G, et al. Intracranial mass lesions: dynamic contrast-enhanced susceptibility-weighted echo-planar perfusion MR imaging. Radiology 2002;223:11-29 CrossRef Medline

11. Thust SC, Heiland S, Falini A, et al. Glioma imaging in Europe: a survey of 220 centres and recommendations for best clinical practice. Eur Radiol 2018;28:3306-17 CrossRef Medline

12. Suh CH, Kim HS, Jung SC, et al. Perfusion MRI as a diagnostic biomarker for differentiating glioma from brain metastasis: a systematic review and meta-analysis. Eur Radiol 2018;28:3819-31 CrossRef Medline

13. Weber MA, Zoubaa S, Schlieter M, et al. Diagnostic performance of spectroscopic and perfusion MRI for distinction of brain tumors. Neurology 2006;66:1899-906 CrossRef Medline 
14. Chiang IC, Kuo YT, Lu CY, et al. Distinction between high-grade gliomas and solitary metastases using peritumoral 3-T magnetic resonance spectroscopy, diffusion, and perfusion imagings. Neuroradiology 2004;46:619-27 CrossRef Medline

15. Law M, Cha S, Knopp EA, et al. High-grade gliomas and solitary metastases: differentiation by using perfusion and proton spectroscopic MR imaging. Radiology 2002;222:715-21 CrossRef Medline

16. Boxerman JL, Schmainda KM, Weisskoff RM. Relative cerebral blood volume maps corrected for contrast agent extravasation significantly correlate with glioma tumor grade, whereas uncorrected maps do not. AJNR Am J Neuroradiol 2006;27:859-67 Medline

17. Sunwoo L, Yun TJ, You SH, et al. Differentiation of glioblastoma from brain metastasis: qualitative and quantitative analysis using arterial spin labeling MR imaging. PLoS One 2016;11:e0166662 CrossRef Medline

18. Lemee JM, Clavreul A, Menei P. Intratumoral heterogeneity in glioblastoma: don't forget the peritumoral brain zone. Neuro Oncol 2015;17:1322-32 CrossRef Medline

19. Gulani V, Calamante F, Shellock FG, et al; International Society for Magnetic Resonance in Medicine. Gadolinium deposition in the brain: summary of evidence and recommendations. Lancet Neurol 2017;16:564-70 CrossRef Medline

20. Kuo PH, Kanal E, Abu-Alfa AK, et al. Gadolinium-based MR contrast agents and nephrogenic systemic fibrosis. Radiology 2007;242:647-49 CrossRef Medline

21. Schieda N, Blaichman JI, Costa AF, et al. Gadolinium-based contrast agents in kidney disease: a comprehensive review and clinical practice guideline issued by the Canadian Association of Radiologists. Can J Kidney Health Dis 2018;5:2054358118778573 CrossRef Medline

22. Bjornerud A, Vatnehol SAS, Larsson C, et al. Signal enhancement of the dentate nucleus at unenhanced MR imaging after very high cumulative doses of the macrocyclic gadolinium-based contrast agent gadobutrol: an observational study. Radiology 2017;285:434-44 CrossRef Medline

23. Hua J, Liu P, Kim T, et al. MRI techniques to measure arterial and venous cerebral blood volume. Neuroimage 2019;187:17-31 CrossRef Medline

24. Hua J, Qin Q, Donahue MJ, et al. Inflow-based vascular-space-occupancy (iVASO) MRI. Magn Reson Med 2011;66:40-56 CrossRef Medline

25. Hua J, Qin Q, Pekar JJ, et al. Measurement of absolute arterial cerebral blood volume in human brain without using a contrast agent. NMR Biomed 2011;24:1313-25 CrossRef Medline

26. Huber L, Goense J, Kennerley AJ, et al. Investigation of the neurovascular coupling in positive and negative BOLD responses in human brain at 7 T. Neuroimage 2014;97:349-62 CrossRef Medline

27. Ito $\mathrm{H}$, Ibaraki M, Kanno I, et al. Changes in the arterial fraction of human cerebral blood volume during hypercapnia and hypocapnia measured by positron emission tomography. J Cereb Blood Flow Metab 2005;25:852-57 CrossRef Medline

28. van Zijl PC, Eleff SM, Ulatowski JA, et al. Quantitative assessment of blood flow, blood volume and blood oxygenation effects in functional magnetic resonance imaging. Nat Med 1998;4:15967 CrossRef Medline

29. Hua J, Unschuld PG, Margolis RL, et al. Elevated arteriolar cerebral blood volume in prodromal Huntington's disease. Mov Disord 2014;29:396-401 CrossRef Medline

30. Hua JL, Blair NIS. Abnormal grey matter arteriolar cerebral blood volume and its association with the presence of $\mathrm{E} 4$ allele of the apolipoprotein E (APOE) gene in elderly subjects at risk for Alzheimer's disease. In: Proceedings of the Scientific Meeting and Exhibition of the International Society for Magnetic Resonance in Medicine, May 7-13, 2016. Singapore; 4030

31. Hua J, Brandt AS, Lee S, et al. Abnormal grey matter arteriolar cerebral blood volume in schizophrenia measured with 3D inflow-based vascular-space-occupancy MRI at 7T. Schizophr Bull 2017;43:620-32 CrossRef Medline
32. Wu Y, Agarwal S, Jones CK, et al. Measurement of arteriolar blood volume in brain tumors using MRI without exogenous contrast agent administration at 7T. J Magn Reson Imaging 2016;44:1244-55 CrossRef Medline

33. Wetzel SG, Cha S, Johnson G, et al. Relative cerebral blood volume measurements in intracranial mass lesions: interobserver and intraobserver reproducibility study. Radiology 2002;224:797-803 CrossRef Medline

34. Ricci R, Bacci A, Tugnoli V, et al. Metabolic findings on 3T 1H-MR spectroscopy in peritumoral brain edema. AJNR Am J Neuroradiol 2007;28:1287-91 CrossRef Medline

35. Cha S, Lupo JM, Chen MH, et al. Differentiation of glioblastoma multiforme and single brain metastasis by peak height and percentage of signal intensity recovery derived from dynamic susceptibility-weighted contrast-enhanced perfusion MR imaging. AJNR Am J Neuroradiol 2007;28:1078-84 CrossRef Medline

36. Cha S. Perfusion MR imaging of brain tumors. Top Magn Reson Imaging 2004;15:279-89 CrossRef Medline

37. Bertossi M, Virgintino D, Maiorano E, et al. Ultrastructural and morphometric investigation of human brain capillaries in normal and peritumoral tissues. Ultrastruct Pathol 1997;21:41-49 CrossRef Medline

38. Kelly PJ, Daumas-Duport C, Scheithauer BW, et al. Stereotactic histologic correlations of computed tomography- and magnetic resonance imaging-defined abnormalities in patients with glial neoplasms. Mayo Clin Proc 1987;62:450-59 CrossRef Medline

39. Daumas-Duport C, Monsaigneon V, Blond S, et al. Serial stereotactic biopsies and CT scan in gliomas: correlative study in 100 astrocytomas, oligo-astrocytomas and oligodendrocytomas. J Neurooncol 1987;4:317-28 CrossRef Medline

40. Server A, Josefsen R, Kulle B, et al. Proton magnetic resonance spectroscopy in the distinction of high-grade cerebral gliomas from single metastatic brain tumors. Acta Radiol 2010;51:316-25 CrossRef Medline

41. Halshtok NO, Sadetzki S, Chetrit A, et al. Perfusion-weighted imaging of peritumoral edema can aid in the differential diagnosis of glioblastoma mulltiforme versus brain metastasis. Isr Med Assoc J 2013;15:103-05 Medline

42. Oei MTH, Meijer FJA, Mordang JJ, et al. Observer variability of reference tissue selection for relative cerebral blood volume measurements in glioma patients. Eur Radiol 2018;28:3902-11 CrossRef Medline

43. Usinskiene J, Ulyte A, Bjornerud A, et al. Optimal differentiation of high- and low-grade glioma and metastasis: a meta-analysis of perfusion, diffusion, and spectroscopy metrics. Neuroradiology 2016;58:339-50 CrossRef Medline

44. Park MJ, Kim HS, Jahng GH, et al. Semiquantitative assessment of intratumoral susceptibility signals using non-contrast-enhanced high-field high-resolution susceptibility-weighted imaging in patients with gliomas: comparison with MR perfusion imaging. AJNR Am J Neuroradiol 2009;30:1402-08 CrossRef Medline

45. Hu LS, Ning S, Eschbacher JM, et al. Radiogenomics to characterize regional genetic heterogeneity in glioblastoma. Neuro Oncol 2017;19:128-37 CrossRef Medline

46. Nguyen TB, Cron GO, Bezzina K, et al. Correlation of tumor immunohistochemistry with dynamic contrast-enhanced and DSC-MRI parameters in patients with gliomas. AJNR Am J Neuroradiol 2016;37:2217-23 CrossRef Medline

47. Meyer M, Reimand J, Lan X, et al. Single cell-derived clonal analysis of human glioblastoma links functional and genomic heterogeneity. Proc Natl Acad Sci USA 2015;112:851-56 CrossRef Medline

48. Stieber D, Golebiewska A, Evers L, et al. Glioblastomas are composed of genetically divergent clones with distinct tumourigenic potential and variable stem cell-associated phenotypes. Acta Neuropathol 2014;127:203-19 CrossRef Medline

49. Jinnouchi T, Shibata S, Fukushima M, et al. Ultrastructure of capillary permeability in human brain tumor, Part 6: metastatic brain 
tumor with brain edema [in Japanese]. No Shinkei Geka 1988;16(5 Suppl):563-68 Medline

50. Sarkaria JN, Hu LS, Parney IF, et al. Is the blood-brain barrier really disrupted in all glioblastomas? A critical assessment of existing clinical data. Neuro Oncol 2018;20:184-91 CrossRef Medline

51. Lai PH, Chung HW, Chang HC, et al. Susceptibility-weighted imaging provides complementary value to diffusion-weighted imaging in the differentiation between pyogenic brain abscesses, necrotic glioblastomas, and necrotic metastatic brain tumors. Eur J Radiol 2019;117:56-61 CrossRef Medline

52. $\mathrm{Fu} \mathrm{JH}$, Chuang TC, Chung $\mathrm{HW}$, et al. Discriminating pyogenic brain abscesses, necrotic glioblastomas, and necrotic metastatic brain tumors by means of susceptibility-weighted imaging. Eur Radiol 2015;25:1413-20 CrossRef Medline

53. Pinker K, Noebauer-Huhmann IM, Stavrou I, et al. High-resolution contrast-enhanced, susceptibility-weighted MR imaging at $3 \mathrm{~T}$ in patients with brain tumors: correlation with positron-emission tomography and histopathologic findings. AJNR Am J Neuroradiol 2007;28:1280-86 CrossRef Medline

54. Park SM, Kim HS, Jahng GH, et al. Combination of high-resolution susceptibility-weighted imaging and the apparent diffusion coefficient: added value to brain tumour imaging and clinical feasibility of non-contrast MRI at 3 T. Br J Radiol 2010;83:466-75 CrossRef Medline

55. Mouthuy N, Cosnard G, Abarca-Quinones J, et al. Multiparametric magnetic resonance imaging to differentiate high-grade gliomas and brain metastases. J Neuroradiol 2012;39:301-07 CrossRef Medline

56. Vallée A, Guillevin C, Wager M, et al. Added value of spectroscopy to perfusion MRI in the differential diagnostic performance of common malignant brain tumors. AJNR Am J Neuroradiol 2018;39:1423-31 CrossRef Medline

57. Ma JH, Kim HS, Rim NJ, et al. Differentiation among glioblastoma multiforme, solitary metastatic tumor, and lymphoma using whole-tumor histogram analysis of the normalized cerebral blood volume in enhancing and perienhancing lesions. AJNR Am J Neuroradiol 2010;31:1699-706 CrossRef Medline

58. Iadecola C, Nedergaard M. Glial regulation of the cerebral microvasculature. Nat Neurosci 2007;10:1369-76 CrossRef Medline

59. Kim T, Hendrich KS, Masamoto K, et al. Arterial versus total blood volume changes during neural activity-induced cerebral blood flow change: implication for BOLD fMRI. J Cereb Blood Flow Metab 2007;27:1235-47 CrossRef Medline

60. Gitiaux C, Kostallari E, Lafuste P, et al. Whole microvascular unit deletions in dermatomyositis. Ann Rheum Dis 2013;72:445-52 CrossRef Medline

61. Shen Z, Lu Z, Chhatbar PY, et al. An artery-specific fluorescent dye for studying neurovascular coupling. Nat Methods 2012;9:273-76 CrossRef Medline

62. Hansen-Smith F, Egginton S, Zhou AL, et al. Growth of arterioles precedes that of capillaries in stretch-induced angiogenesis in skeletal muscle. Microvasc Res 2001;62:1-14 CrossRef Medline

63. Zhao JM, Clingman CS, Narvainen MJ, et al. Oxygenation and hematocrit dependence of transverse relaxation rates of blood at $3 \mathrm{~T}$. Magn Reson Med 2007;58:592-97 CrossRef Medline

64. Vanzetta I, Hildesheim R, Grinvald A. Compartment-resolved imaging of activity-dependent dynamics of cortical blood volume and oximetry. J Neurosci 2005;25:2233-44 CrossRef

65. Hillman EM, Devor A, Bouchard MB, et al. Depth-resolved optical imaging and microscopy of vascular compartment dynamics during somatosensory stimulation. Neuroimage 2007;35:89-104 CrossRef Medline

66. Gunther M, Bock M, Schad LR. Arterial spin-labeling in combination with a look-locker sampling strategy: inflow turbo-sampling EPI-FAIR (ITS-FAIR). Magn Reson Med 2001;46:974-84 CrossRef Medline

67. Warmuth C, Gunther M, Zimmer C. Quantification of blood flow in brain tumors: comparison of arterial spin labeling and dynamic susceptibility-weighted contrast-enhanced MR imaging. Radiology 2003;228:523-32 CrossRef Medline

68. Boxerman JL, Prah DE, Paulson ES, et al. The role of preload and leakage correction in gadolinium-based cerebral blood volume estimation determined by comparison with MION as a criterion standard. AJNR Am J Neuroradiol 2012;33:1081-87 CrossRef Medline

69. Welker K, Boxerman J, Kalnin A, et al; American Society of Functional Neuroradiology MR Perfusion Standards and Practice Subcommittee of the ASFNR Clinical Practice Committee. ASFNR recommendations for clinical performance of MR dynamic susceptibility contrast perfusion imaging of the brain. AJNR Am J Neuroradiol 2015;36:E41-51 CrossRef Medline 